\title{
Groundwater-quality Assessment Models with Total Nitrogen Transformation Effects
}

\author{
Supawan Yena ${ }^{1,2, *}$, Nopparat Pochai ${ }^{1,2}$ \\ ${ }^{1}$ Department of Mathematics, Faculty of Science, King Mongkut's Institute of Technology Ladkrabang, Bangkok 10520, Thailand \\ ${ }^{2}$ Centre of Excellence in Mathematics, Commission on Higher Education(CHE), Si Ayutthaya Road, Bangkok 10400, Thailand
}

Received November 29, 2019; Revised February 1, 2020; Accepted February 7, 2020

Copyright $@ 2020$ by authors, all rights reserved. Authors agree that this article remains permanently open access under the terms of the Creative Commons Attribution License 4.0 International License

\begin{abstract}
Nitrogen is emitted extensively by industrial companies, increasing nitrogen compounds such as ammonia, nitrate, and nitrite in soil and water as a result of nitrogen cycle reactions. Groundwater contamination with nitrates and nitrites impacts human health. Mathematical models can explain groundwater contamination with nitrates and nitrites. Hydraulic head model provides the hydraulic head of groundwater. Groundwater velocity model provided x- and $\mathrm{y}$ - direction vector in groundwater. Groundwater contamination distribution model provides nitrogen, nitrate and nitrite concentration. Finite difference techniques are approximate the models solution. Alternating direction explicit method was used to clarify hydraulic head model. Centered space explained groundwater velocity model. Forward time central space was used to predict groundwater transportation of contamination models. We simulate different circumstances to explain the pollution in leachate water underground, paying attention to the toxic nitrogen, ammonia, nitrate, nitrite blended in the water.
\end{abstract}

Keywords Finite Difference Method, Alternating Direction Explicit Method, Centred Space Tecnique, Forward Time Centred Space

\section{Introduction}

Water pollution is a worldwide issue created by development of population and economic growth. Nitrogen (N2) is a critical nutrients for all living organisms. Human activity has a serious effect on the natural nitrogen cycle, particularly due to the development of industrial fertilizer production. Nitrogen is emitted extensively by industrial companies, increasing nitrogen compounds, such as ammonia $\left(\mathrm{NH}_{3}\right)$, nitrate $\left(\mathrm{NO}_{3}^{-}\right)$, and nitrite $\left(\mathrm{NO}_{2}^{-}\right)$in soil and water, as a result of nitrogen cycle reactions in Fig 1.

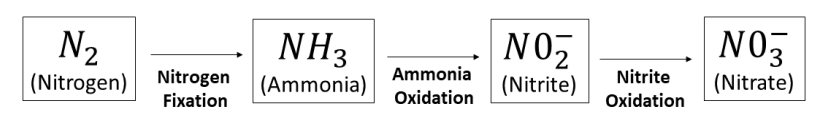

Figure 1. Nitrogen Cycle

Microorganisms are important as converters of soil ammonia into nitrates, in the nitrogen cycle, which can be used by plants. The cycle of nitrification allows two separate groups: bacteria that convert ammonia into nitrites and bacteria that convert nitrites into nitrates. Surface water leaches nitrogen from soil to groundwater from waste waters and the oxidation of nitrogen waste from humans and other animals. In agricultural areas, ammonia in the water supply enters the body by coughing, chewing or contact with the skin: ammonia enters the body and reacts with water to form ammonium hydroxide, which is very corrosive and on contact can destroy body cells. Ammonia is not often present in groundwater, because it is converted into nitrates by soil bacteria, by aerobic bacteria (nitrification,) using oxygen. Nitrosomonas bacteria first convert nitrogen to nitrites and subsequently nitrobacter convert nitrites to nitrates, a plant nutrient. Dietary nitrate is converted to nitrite through a non-enzymatic process and nitric oxide $(N O)$ by symbiotic bacteria in the oral cavity and stomach, which then forms several compounds with different effects in the body. Nitrates and nitrites are naturally found in vegetables, but also added as a preservative to some processed foods, for example, bacon. Nitrates or nitrites in water contaminates drinking water and impacts human health by decreasing blood cell ability to carry oxygen, resulting in the oxygen deficiency in some body parts, that can be linked to blue baby syndrome[1]. Nitrates have a carcinogenic nitrosamine, that has caused esophagus, stomach, or colon cancer, and other illnesses [2]. Nitrite also reacts with amines and amides, present in proteins from the diet or from medications, to form $\mathrm{N}$ nitrosamines and $\mathrm{N}$ nitrosamides. These compounds damage DNA and cause birth 
cancers[3]. There are many dangers of nitrates and nitrites, but we can prevent amounts of them, from exceeding the standards by management based on mathematical models. Yamashita and Sugio (2000) developed a model of advection dispersion and biochemical reactions, considering heterotrophic processes [4]. Dehghan (2004) solved the one-dimensional advection diffusion equation with constant coefficients by two-level finite difference approximation techniques, developed by Warming and Hyett [5]. Gardenas (2005) described a two-dimensional modeling approach to improve fertigation strategies and soil types on nitrate leaching potential [6]. Zhiqiang (2012) presented a novel Best Management Practices (BMP) device for highway and urban stormwater treatment with emphasis on numerical modeling, called a First Flush Reactor (FFR). Stormwater runoff is a common non point source of water pollution in rivers, lakes, estuaries and coastal beaches. While most pollutants and nutrients, including nitrate nitrogen, in stormwater are discharged into receiving waters during the first-flush period, no existing BMP have been specifically designed to capture and treat the first-flush portion of urban stormwater runoff [7]. Pochai and Kraychang (2016) generated two mathematical models for simulating water pollutant level and pollution control in a connected reservoir system [8]. Kewalee and Pochai (2018) explained the governing equation in the air quality model in three-dimensional advection-diffusion equations with time dependence [9].

Here, we measured nitrogen dispersion on total nitrogen transformation effects models. First, to supply the groundwater head, we used a hydraulic head model, followed by a groundwater velocity model based on vectors in the $\mathrm{x}$ - and $\mathrm{y}$ directions. Next, the model was used to define nitrogen, ammonia, nitrate, nitrite for the allocation of groundwater contamination. A finite element technique was used to find responses. An alternating direction explicit method was used to clarify hydraulic head model. Centered space technique explained groundwater velocity model. Forward time central space technique was used to predict nitrogen dispersion models. Nitrogen enters groundwater at varying concentrations and in varying forms. We simulated various conditions to explain the pollution in subterranean leachate water, considering the toxic nitrogen, ammonia, nitrates and nitrites mixed in water.

\section{Nitrogen Pollutant Measure Models}

\subsection{A Two Dimensional Hydraulic Head Model}

Drinking groundwater can be hazardous to health, especially for infants and pregnant women. If there is excessive rainfall or over irrigation, nitrate will be leached below the plant's root zone and may eventually reach groundwater [10]. Darcy's Law defines the relationship between groundwater flow rate. In the steady state, we used Darcy's law[12]

$$
K\left(\frac{\partial^{2} h}{\partial x^{2}}+\frac{\partial^{2} h}{\partial y^{2}}\right) \pm W=S \frac{\partial h}{\partial t} .
$$

where $h(x, y, 0)(\mathrm{m})$ is the hydraulic head, $K(\mathrm{~m} /$ day $)$ is the hydraulic conductivity in homogeneous aquifer, $W\left(d a y^{-1}\right)$ is the source or sink function, $S$ is the specific storage coefficient for all $(x, y) \in \Omega \times[0, T]$.

The initial condition is a mean potential hydraulic head:

$$
h(x, y, 0)=h_{0}
$$

for all $(x, y)$ in the considered area $(\Omega)$

$$
\Omega=[0, I] \times[0, J]
$$

when $I$ is the width and $J$ is the length.

The assumed boundary conditions are

$$
\begin{aligned}
& \frac{\partial h}{\partial y}(x, J, t)=N_{h}, \text { for all } x \in[0, I], t>0, \\
& \frac{\partial h}{\partial y}(x, 0, t)=S_{h}, \text { for all } x \in[0, I], t>0, \\
& \frac{\partial h}{\partial x}(0, y, t)=W_{h}, \text { for all } y \in[0, J], t>0, \\
& \frac{\partial h}{\partial x}(I, y, t)=E_{h}, \text { for all } y \in[0, J], t>0,
\end{aligned}
$$

where $N_{h}, S_{h}, W_{h}$ and $E_{h}$ are the rates of change of hydraulic head on the north, south, west and east domain boundaries.

\subsection{A Two Dimensional Groundwater Flow Velocity Model}

Hydraulic head becomes velocity potential in this model,

$$
\begin{aligned}
& u=-\frac{\partial h}{\partial x}, \\
& v=-\frac{\partial h}{\partial y},
\end{aligned}
$$

where $u, v(\mathrm{~m} /$ day) are groundwater flow velocity in $x$ - and $y$ directions, respectively.

\subsection{Nitrogen Compound Dispersion Models}

Leachate water infiltrates into the ground; it penetrates soil pores and becomes groundwater. Nitrogen enters groundwater at varying concentrations and in varying forms. Nitrogen is an important water contaminant. Microorganisms are important, as, in the nitrogen cycle. An advection-diffusion model provides a continuous description of groundwater pollutant transport. The models are used to describe amount of toxic substances in groundwater on a two-dimensional vertically averaged groundwater pollution dispersion model[11].

\subsubsection{A total nitrogen dispersion model}

The pollutant concentration measurement of total nitrogen (TN) in surface water is based on a two-dimensional advection diffusion reaction equation.

$$
\frac{\partial \phi}{\partial t}+u \frac{\partial \phi}{\partial x}+v \frac{\partial \phi}{\partial y}=D_{0 x} \frac{\partial^{2} \phi}{\partial x^{2}}+D_{0 y} \frac{\partial^{2} \phi}{\partial y^{2}}+W_{s}
$$

where $\phi(x, y, t)\left(\mathrm{kg} / \mathrm{m}^{3}\right)$ is total nitrogen pollutant concentration of groundwater, $u$ and $v$ (m/day) are the $x-$ the $y-$ 
direction groundwater flow velocity, $D_{0 x}$ and $D_{0 y}\left(\mathrm{~m}^{2} /\right.$ day $)$ are the $x$ - the $y$ - diffusion coefficient of groundwater pollution through specified soil and $W_{s}\left(d a y^{-1}\right)$ is groundwater pollutant sources function.

\section{Initial condition of total nitrogen dispersion model}

Total nitrogen dispersion is described with the conditions in the following sections, where the potential groundwater pollutant concentration in the consider area is described by

$$
\phi(x, y, 0)=f_{\phi}(x, y) \text { for all }(x, y) \in \Omega
$$

where $f_{\phi}(x, y)$ is a averaged potential total nitrogen concentration in the considered area.

\section{Boundary condition of total nitrogen dispersion model}

The rates of change of the pollutant concentration along the domain boundaries are assumed to be:

$$
\begin{aligned}
& \frac{\partial \phi}{\partial y}(x, J, t)=N_{\phi}, \text { for all } x \in[0, I], t>0, \\
& \frac{\partial \phi}{\partial y}(x, 0, t)=S_{\phi}, \text { for all } x \in[0, I], t>0, \\
& \frac{\partial \phi}{\partial x}(0, y, t)=W_{\phi}, \text { for all } y \in[0, J], t>0, \\
& \frac{\partial \phi}{\partial x}(I, y, t)=E_{\phi}, \text { for all } y \in[0, J], t>0,
\end{aligned}
$$

where $N_{\phi}, S_{\phi}, W_{\phi}$ and $E_{\phi}$ are the rates of change of the total nitrogen concentration on the north, south, west and east domain boundaries.

\subsubsection{An ammonia dispersion model}

The total nitrogen transformed to be the ammonia. The ammonia dispersion model is described by

$$
\frac{\partial \psi}{\partial t}+u \frac{\partial \psi}{\partial x}+v \frac{\partial \psi}{\partial y}=D_{1 x} \frac{\partial^{2} \psi}{\partial x^{2}}+D_{1 y} \frac{\partial^{2} \psi}{\partial y^{2}}+R_{0} \phi
$$

where $\psi(x, y, t)\left(\mathrm{kg} / \mathrm{m}^{3}\right)$ is ammonia pollutant concentration of groundwater, $D_{1 x}$ and $D_{1 y}\left(\mathrm{~m}^{2} /\right.$ day) are the $x-$ and $y$ - diffusion coefficient of ammonia pollution through specified soil, $R_{0}$ is transformed nitrogen rate.

\section{Initial condition of ammonia dispersion model}

Dispersion of ammonia with following conditions, If the potential ammonia concentration in the consider area is described by

$$
\psi(x, y, 0)=f_{\psi}(x, y) \text { for all }(x, y) \in \Omega
$$

where $f \psi(x, y)$ is a averaged potential ammonia pollutant concentration in the considered area.

\section{Boundary condition of ammonia dispersion model}

The rates of change of the pollutant concentration along the domain boundaries are assumed by

$$
\begin{aligned}
& \frac{\partial \psi}{\partial y}(x, J, t)=N_{\psi}, \text { for all } x \in[0, I], t>0, \\
& \frac{\partial \psi}{\partial y}(x, 0, t)=S_{\psi}, \text { for all } x \in[0, I], t>0, \\
& \frac{\partial \psi}{\partial x}(0, y, t)=W_{\psi}, \text { for all } y \in[0, J], t>0, \\
& \frac{\partial \psi}{\partial x}(I, y, t)=E_{\psi}, \text { for all } y \in[0, J], t>0,
\end{aligned}
$$

Similar, where $N_{\psi}, S_{\psi}, W_{\psi}$ and $E_{\psi}$ are the rates of change of the ammonia concentration on the north, south, west and east domain boundaries.

\subsubsection{A nitrite dispersion model}

The model of nitrite dispersion can be described by

$$
\frac{\partial \xi}{\partial t}+u \frac{\partial \xi}{\partial x}+v \frac{\partial \xi}{\partial y}=D_{2 x} \frac{\partial^{2} \xi}{\partial x^{2}}+D_{2 y} \frac{\partial^{2} \xi}{\partial y^{2}}+R_{1} \psi
$$

where $\xi(x, y, t)\left(\mathrm{kg} / \mathrm{m}^{3}\right)$ is nitrite pollutant concentration of groundwater, $D_{2 x}$ and $D_{2 y}\left(\mathrm{~m}^{2} /\right.$ day) are the $x-$ and $y-$ diffusion coefficient of nitrite pollution through specified soil, $R_{1}$ is ammonia transformed rate.

\section{Initial condition of nitrite dispersion}

Dispersion of nitrite with following conditions, If the potential nitrite pollutant concentration in the consider area is described by

$$
\xi(x, y, 0)=f_{\xi}(x, y) \text { for all }(x, y) \in \Omega .
$$

where $f_{\xi}(x, y)$ is a averaged potential nitrite pollutant concentration in the considered area.

\section{Boundary condition of nitrite dispersion}

Similarly, the rates of change are assumed by $N_{\xi}, S_{\xi}, W_{\xi}$ and $E_{\xi}$.

\subsubsection{A nitrate dispersion model}

The model of nitrate dispersion can be described by

$$
\frac{\partial \zeta}{\partial t}+u \frac{\partial \zeta}{\partial x}+v \frac{\partial \zeta}{\partial y}=D 3_{x} \frac{\partial^{2} \zeta}{\partial x^{2}}+D 3_{y} \frac{\partial^{2} \zeta}{\partial y^{2}}+R_{2} \xi
$$

where $\zeta(x, y, t)\left(\mathrm{kg} / \mathrm{m}^{3}\right)$ is nitrate pollutant concentration of groundwater, $D_{3 x}$ and $D_{3 y}\left(\mathrm{~m}^{2} /\right.$ day $)$ are the $x-$ and $y-$ the diffusion coefficient of nitrate through specified soil, $R_{2}$ is the transformed nitrite rate. 


\section{Initial condition of nitrate dispersion}

Dispersion of nitrite with following conditions, If the potential nitrate pollutant concentration in the consider area is described by

$$
\zeta(x, y, 0)=f_{\zeta}(x, y) \text { for all }(x, y) \in \Omega .
$$

where $f_{\zeta(x, y)}$ is a averaged potential nitrite pollutant concentration in the considered area.

\section{Boundary condition of nitrate dispersion}

Similarly, the rates of change are assumed by $N_{\zeta}, S_{\zeta}, W_{\zeta}$ and $E_{\zeta}$.

\section{Numerical Techniques for The Mod- els}

A numerical solution is based on partial differential equation can solving by finite difference technique. A two-dimension hydraulic head model under several boundary conditions with modified alternating direction explicit method (ADEMM). A two-dimension groundwater velocity model with centered space (CS). Nitrogen dispersion models with forward time central space (FTCS).

We discretize the domain by dividing the interval $[0, I]$ into $M$ subintervals, so that $M \Delta x=I$ similarly divide $[0, J]$ and $[0, T]$ into $N$ and $P$ subintervals, $N \Delta y=J$ and $P \Delta t=T$. The value of the difference approximation at point $x_{i}=i \Delta x$, $y_{j}=j \Delta y$ and $t_{n}=n \Delta t$, when $0 \leq i \leq M, 0 \leq j \leq N$ and $0 \leq n \leq P$, where $M, N$ and $P$ are positive integers.

\subsection{Modified alternating direction explicit method (ADEMM) for the hydraulic head model}

We introduce the modified alternating direction explicit method to a two-dimensional vertically averaged groundwater flow model ( 1 ).

1. Initialization setting that

$$
\begin{aligned}
& h 1_{i, j}^{n}=h 1_{i, j}^{n} \\
& h 2_{i, j}^{n}=h 2_{i, j}^{n}
\end{aligned}
$$

2. First stage setting that

$$
\begin{aligned}
\frac{\partial h}{\partial t} & \approx \frac{h_{i, j}^{n+1}-h_{i, j}^{n}}{\Delta t}, \\
\frac{\partial^{2} h}{\partial x^{2}} & \approx \frac{h 1_{i-1, j}^{n+1}-h 1_{i, j}^{n+1}-h 1_{i, j}^{n}+h 1_{i+1, j}^{n}}{(\Delta x)^{2}} \\
\frac{\partial^{2} h}{\partial y^{2}} & \approx \frac{h 1_{i, j-1}^{n+1}-h 1_{i, j}^{n+1}-h 1_{i, j}^{n}+h 1_{i, j+1}^{n}}{(\Delta y)^{2}} .
\end{aligned}
$$

Substituting ( 28$)-(30)$ into ( 1 ), we obtain

$$
\begin{aligned}
& K x_{i+\frac{1}{2}, j}\left(\frac{h 1_{i+1, j}^{n}-h 1_{i, j}^{n}}{(\Delta x)^{2}}\right)+K x_{i-\frac{1}{2}, j}\left(\frac{h 1_{i-1, j}^{n+1}-h 1_{i, j}^{n+1}}{(\Delta x)^{2}}\right) \\
& +K y_{i, j+\frac{1}{2}}\left(\frac{h 1_{i, j+1}^{n}-h 1_{i, j}^{n}}{(\Delta y)^{2}}\right)+K y_{i, j-\frac{1}{2}}\left(\frac{h 1_{i, j-1}^{n+1}-h 1_{i, j}^{n+1}}{(\Delta y)^{2}}\right) \\
& \pm W=S\left(\frac{h 1_{i, j}^{n+1}-h 1_{i, j}^{n}}{\Delta t}\right) .
\end{aligned}
$$

Then the explicit finite difference equation becomes

$$
\begin{aligned}
& h 1_{i, j}^{n+1}=\frac{1}{1+\alpha_{2}+\beta_{2}}\left(\alpha_{2} h 1_{i-1, j}^{n+1}+\beta_{2} h 1_{i, j-1}^{n+1}\right. \\
& \left.+\alpha_{1} h 1_{i+1, j}^{n}+\beta_{1} h 1_{i, j+1}^{n}-\left(\alpha_{1}+\beta_{1}-1\right) h 1_{i, j}^{n}\right) \\
& +\gamma .
\end{aligned}
$$

where $\alpha_{1}=\frac{K x_{i+\frac{1}{2}, j} \Delta t}{S(\Delta x)^{2}}, \alpha_{2}=\frac{K x_{i-\frac{1}{2}, j} \Delta t}{S(\Delta x)^{2}}$,

$$
\beta_{1}=\frac{K y_{i, j+\frac{1}{2}} \Delta t}{S(\Delta y)^{2}}, \beta_{2}=\frac{K y_{i, j-\frac{1}{2}} \Delta t}{S(\Delta y)^{2}} \text { and } \gamma=\frac{W \Delta t}{S} \text {. }
$$

3. Second stage setting that

$$
\begin{aligned}
\frac{\partial h}{\partial t} & \approx \frac{h_{i, j}^{n+1}-h_{i, j}^{n}}{\Delta t}, \\
\frac{\partial^{2} h}{\partial x^{2}} & \approx \frac{h 2_{i+1, j}^{n+1}-h 2_{i, j}^{n+1}-h 2_{i, j}^{n}+h 2_{i-1, j}^{n}}{(\Delta x)^{2}}, \\
\frac{\partial^{2} h}{\partial y^{2}} & \approx \frac{h 2_{i, j+1}^{n+1}-h 2_{i, j}^{n+1}-h 2_{i, j}^{n}+h 2_{i, j-1}^{n}}{(\Delta y)^{2}} .
\end{aligned}
$$

Substituting ( 33 ) - ( 35 ) into ( 1 ), we obtain

$$
\begin{aligned}
& K x_{i-\frac{1}{2}, j}\left(\frac{h 2_{i-1, j}^{n}-h 2_{i, j}^{n}}{(\Delta x)^{2}}\right)+K x_{i+\frac{1}{2}, j}\left(\frac{h 2_{i+1, j}^{n+1}-h 2_{i, j}^{n+1}}{(\Delta x)^{2}}\right) \\
& +K y_{i, j-\frac{1}{2}}\left(\frac{h 2_{i, j-1}^{n}-h 2_{i, j}^{n}}{(\Delta y)^{2}}\right)+K y_{i, j+\frac{1}{2}}\left(\frac{h 2_{i, j+1}^{n+1}-h 2_{i, j}^{n+1}}{(\Delta y)^{2}}\right) \\
& \pm W=S\left(\frac{h 2_{i, j}^{n+1}-h 2_{i, j}^{n}}{\Delta t}\right) .
\end{aligned}
$$

Then the explicit finite difference equation becomes

$$
\begin{aligned}
& h 2_{i, j}^{n+1}=\frac{1}{1+\alpha_{2}+\beta_{2}}\left(\alpha_{2} h 2_{i+1, j}^{n+1}+\beta_{2} h 2_{i, j+1}^{n+1}\right. \\
& \left.+\alpha_{1} h 2_{i-1, j}^{n}+\beta_{1} h 2_{i, j-1}^{n}-\left(\alpha_{1}+\beta_{1}-1\right) h 2_{i, j}^{n}\right) \\
& +\gamma .
\end{aligned}
$$

4. Last stage setting that

$$
h_{i, j}^{n}=\frac{h 1_{i, j}^{n}+h 2_{i, j}^{n}}{2},
$$

for all $0 \leq i \leq M, 0 \leq j \leq N$ and $0 \leq n \leq P$.

The forward space technique is used to approximate fictitious points on the boundaries solution such as,

$$
\begin{array}{r}
h_{-1, j}^{n}=h_{0, j}^{n}-W_{h} \Delta x, \\
h_{M+1, j}^{n}=h_{M, j}^{n}+E_{h} \Delta x, \\
h_{i,-1}^{n}=h_{i, 0}^{n}-S_{h} \Delta y, \\
h_{i, N+1}^{n}=h_{i, N}^{n}+N_{h} \Delta y .
\end{array}
$$




\subsection{Centered Space Technique for The Ground- water Flow Velocity Model}

We introduced the centered space method to the velocity potential model in two-dimension velocity field Eqs. ( 8 ) - ( 9 ), we have

$$
\begin{aligned}
& u_{i, j}^{n} \approx-\frac{\widehat{h}_{i+1, j}^{n}-\widehat{h}_{i-1, j}^{n}}{2 \Delta x}, \\
& v_{i, j}^{n} \approx-\frac{\widehat{h}_{i, j+1}^{n}-\widehat{h}_{i, j-1}^{n}}{2 \Delta y},
\end{aligned}
$$

where $\widehat{h}$ is the approximated hydraulic head, $\widehat{h}_{i, j}^{n} \approx h_{i, j}^{n}$, for all $0 \leq i \leq I, 0 \leq j \leq J$ and $0 \leq n \leq P$

The centered space technique is used to approximate fictitious points on the boundaries solution such as,

$$
\begin{array}{r}
u_{-1, j}^{n}=u_{1, j}^{n}-2 W_{h} \Delta x, \\
u_{M+1, j}^{n}=u_{M-1, j}^{n}+2 E_{h} \Delta x, \\
v_{i,-1}^{n}=v_{i, 1}^{n}-2 S_{h} \Delta y, \\
v_{i, N+1}^{n}=v_{i, N-1}^{n}+2 N_{h} \Delta y .
\end{array}
$$

\subsection{Forward Time Central Space (FTCS) for Nitrogen Compound Dispersion Models}

The forward time central space method is based on central difference in space and the forward euler method in time, giving first-order convergence in time and second-order convergence in space.

$$
\begin{aligned}
p(x, y, t) & \approx \widehat{p}\left(x_{i \Delta x}, y_{j \Delta y}, t_{n \Delta t}\right)=p_{i, j}^{n} \\
\frac{\partial p}{\partial t} & \approx \frac{p_{i, j}^{n+1}-p_{i, j}^{n}}{\Delta t}, \\
\frac{\partial p}{\partial x} & \approx \frac{p_{i+1, j}^{n}-p_{i-1, j}^{n}}{2 \Delta x}, \\
\frac{\partial p}{\partial y} & \approx \frac{p_{i, j+1}^{n}-p_{i, j-1}^{n}}{2 \Delta y}, \\
\frac{\partial^{2} p}{\partial x^{2}} & \approx \frac{p_{i+1, j}^{n}-2 p_{i, j}^{n}+p_{i-1, j}^{n}}{(\Delta x)^{2}} \\
\frac{\partial^{2} p}{\partial y^{2}} & \approx \frac{p_{i, j+1}^{n}-2 p_{i, j}^{n}+p_{i, j-1}^{n}}{(\Delta y)^{2}} .
\end{aligned}
$$

Taking the forward time centered space technique ( 50 ) - ( 54 ) in nitrogen dispersion models,

An explicit the forward time centered space method for a total nitrogen model

$$
\begin{aligned}
\phi_{i, j}{ }^{n+1} & =\left(\frac{\lambda_{i, j}^{n}}{2}+\varphi_{0}\right) \phi_{i-1, j}{ }^{n}+\left(1-2 \varphi_{0}-2 \Phi_{0}\right) \phi_{i, j}{ }^{n} \\
& +\left(-\frac{\lambda_{i, j}^{n}}{2}+\varphi_{0}\right) \phi_{i+1, j}{ }^{n}+\left(-\frac{\eta_{i, j}^{n}}{2}+\Phi_{0}\right) \phi_{i, j+1}{ }^{n} \\
& +\left(\frac{\eta_{i, j}^{n}}{2}+\Phi_{0}\right) \phi_{i, j-1}{ }^{n}+\varrho
\end{aligned}
$$

where $\lambda_{i, j}^{n}=\frac{u_{i, j}^{n} \Delta t}{\Delta x}, \eta_{i, j}^{n}=\frac{v_{i, j}^{n} \Delta t}{\Delta y}, \varphi_{0}=\frac{D_{0 x} \Delta t}{(\Delta x)^{2}}$,

$\Phi_{0}=\frac{D_{0 y} \Delta t}{(\Delta y)^{2}}$ and $\varrho=\Delta t W_{s}$.

The forward space technique is used to approximate fictitious points on the boundaries solution such as,

$$
\begin{array}{r}
\phi_{-1, j}^{n}=\phi_{0, j}^{n}-W_{\phi} \Delta x, \\
\phi_{M+1, j}^{n}=\phi_{M, j}^{n}+E_{\phi} \Delta x, \\
\phi_{i,-1}^{n}=\phi_{i, 0}^{n}-S_{\phi} \Delta y, \\
\phi_{i, N+1}^{n}=\phi_{i, N}^{n}+N_{\phi} \Delta y .
\end{array}
$$

An explicit the forward time centered space method for an ammonia

$$
\begin{aligned}
\psi_{i, j}{ }^{n+1} & =\left(\frac{\lambda_{i, j}^{n}}{2}+\varphi_{1}\right) \psi_{i-1, j}{ }^{n}+\left(1-2 \varphi_{1}-2 \Phi_{1}\right) \psi_{i, j}{ }^{n} \\
& +\left(-\frac{\lambda_{i, j}^{n}}{2}+\varphi_{1}\right) \psi_{i+1, j}{ }^{n}+\left(-\frac{\eta_{i, j}^{n}}{2}+\Phi_{1}\right) \psi_{i, j+1}{ }^{n} \\
& +\left(\frac{\eta_{i, j}^{n}}{2}+\Phi_{1}\right) \psi_{i, j-1}{ }^{n}+\kappa_{0} \phi
\end{aligned}
$$

where $\varphi_{1}=\frac{D_{1 x} \Delta t}{(\Delta x)^{2}}, \Phi_{1}=\frac{D_{1 y} \Delta t}{(\Delta y)^{2}}$, and $\kappa_{0}=\Delta t R_{0}$.

The forward space technique is used to approximate fictitious points on the boundaries solution such as,

$$
\begin{array}{r}
\psi_{-1, j}^{n}=\psi_{0, j}^{n}-W_{\psi} \Delta x, \\
\psi_{M+1, j}^{n}=\psi_{M, j}^{n}+E_{\psi} \Delta x, \\
\psi_{i,-1}^{n}=\psi_{i, 0}^{n}-S_{\psi} \Delta y, \\
\psi_{i, N+1}^{n}=\psi_{i, N}^{n}+N_{\psi} \Delta y .
\end{array}
$$

An explicit the forward time centered space method for a nitrite

$$
\begin{aligned}
\xi_{i, j}{ }^{n+1} & =\left(\frac{\lambda_{i, j}^{n}}{2}+\varphi_{2}\right) \xi_{i-1, j}{ }^{n}+\left(1-2 \varphi_{2}-2 \Phi_{2}\right) \xi_{i, j}{ }^{n} \\
& +\left(-\frac{\lambda_{i, j}^{n}}{2}+\varphi_{2}\right) \xi_{i+1, j}{ }^{n}+\left(-\frac{\eta_{i, j}^{n}}{2}+\Phi_{2}\right) \xi_{i, j+1}{ }^{n} \\
& +\left(\frac{\eta_{i, j}^{n}}{2}+\Phi_{2}\right) \xi_{i, j-1}{ }^{n}+\kappa_{1} \psi
\end{aligned}
$$

where $\varphi_{2}=\frac{D_{2 x} \Delta t}{(\Delta x)^{2}}, \Phi_{2}=\frac{D_{2 y} \Delta t}{(\Delta y)^{2}}$, and $\kappa_{1}=\Delta t R_{1}$

The forward space technique is used to approximate fictitious points on the boundaries solution such as,

$$
\begin{array}{r}
\xi_{-1, j}^{n}=\xi_{0, j}^{n}-W_{\xi} \Delta x, \\
\xi_{M+1, j}^{n}=\xi_{M, j}^{n}+E_{\xi} \Delta x, \\
\xi_{i,-1}^{n}=\xi_{i, 0}^{n}-S_{\xi} \Delta y, \\
\xi_{i, N+1}^{n}=\xi_{i, N}^{n}+N_{\xi} \Delta y .
\end{array}
$$


a nitrate

$$
\begin{aligned}
\zeta_{i, j}{ }^{n+1} & =\left(\frac{\lambda_{i, j}^{n}}{2}+\varphi_{3}\right) \zeta_{i-1, j}{ }^{n}+\left(1-2 \varphi_{3}-2 \Phi_{3}\right) \zeta_{i, j}{ }^{n} \\
& +\left(-\frac{\lambda_{i, j}^{n}}{2}+\varphi_{3}\right) \zeta_{i+1, j}{ }^{n}+\left(-\frac{\eta_{i, j}^{n}}{2}+\Phi_{3}\right) \zeta_{i, j+1}{ }^{n} \\
& +\left(\frac{\eta_{i, j}^{n}}{2}+\Phi_{3}\right) \zeta_{i, j-1}{ }^{n}+\kappa_{2} \xi
\end{aligned}
$$

where $\varphi_{3}=\frac{D_{3 x} \Delta t}{(\Delta x)^{2}}, \Phi_{3}=\frac{D_{3 y} \Delta t}{(\Delta y)^{2}}$, and $\kappa_{2}=\Delta t R_{2}$.

The forward space technique is used to approximate fictitious points on the boundaries solution such as,

$$
\begin{array}{r}
\zeta_{-1, j}^{n}=\zeta_{0, j}^{n}-W_{\zeta} \Delta x, \\
\zeta_{M+1, j}^{n}=\zeta_{M, j}^{n}+E_{\zeta} \Delta x, \\
\zeta_{i,-1}^{n}=\zeta_{i, 0}^{n}-S_{\zeta} \Delta y, \\
\zeta_{i, N+1}^{n}=\zeta_{i, N}^{n}+N_{\zeta} \Delta y .
\end{array}
$$

\section{Numerical Experiment}

A two-dimension hydraulic head model provides hydraulic head. Then the calculated results of the model will be input into A two-dimension groundwater velocity model which provides groundwater flow velocity. Next, input the velocity into nitrogen dispersion models that provide nitrogen, ammonia, nitrite and nitrate concentration

\subsection{Numerical simulation of nitrogen dispersion}

The application of nitrogen dispersion models defined grid space $\Delta x=0.5(\mathrm{~m})$ and $\Delta y=0.5(\mathrm{~m})$ time $\Delta t=0.1$ (day) increments in area $10 \times 10\left(\mathrm{~m}^{2}\right)$. The first model, considering the hydraulic conductivity in homogeneous aquifer $K=0.15$ (m/day), the specific storage coefficient $S=10$, source function $W=0.1\left(\mathrm{~d} a y^{-1}\right)$. The initial $h_{0}=30(\mathrm{~m})$ and boundary conditions $N_{h}=0.001, S_{h}=-0.001, W_{h}=-0.001$ and $E_{h}=0$. It mean that water flows to north, south and west on $\Omega$ domain. Consider the hydraulic head of groundwater by using the modified alternating direction explicit method approximate solution as shown in Fig 2 - Fig 3.

The second model, input hydraulic head in first model to approximate groundwater velocity in ( 8 ) and (9) by using CS. The direction of groundwater in consider area as shown in Fig 4.

Next, input groundwater to approximate nitrogen dispersion. Organic material is transformed into ammonia, then into nitrite, and lastly into nitrate by using FTCS, considering diffusion coefficient of nitrogen $D_{0 x}=D_{0 y}=0.002$ and source function of pollution through specified $W s=0.001 * W$. The initial $\phi_{0}=2 \mathrm{~m}$ and boundary conditions $N_{\phi}=0.001 N_{h}$, $S_{\phi}=-0.001 S_{h}, W_{\phi}=-0.001 W_{h}$ and $E_{\phi}=0.001 E_{h}$. It mean that water flows to north, south and west on $\Omega$ domain. The approximate solution as shown in Fig 5.

Considering diffusion coefficient of ammonia $D_{1 x}=$ $D_{1 y}=0.0013$ and rate of nitrogen dispersion $R_{0}=0.00005$.

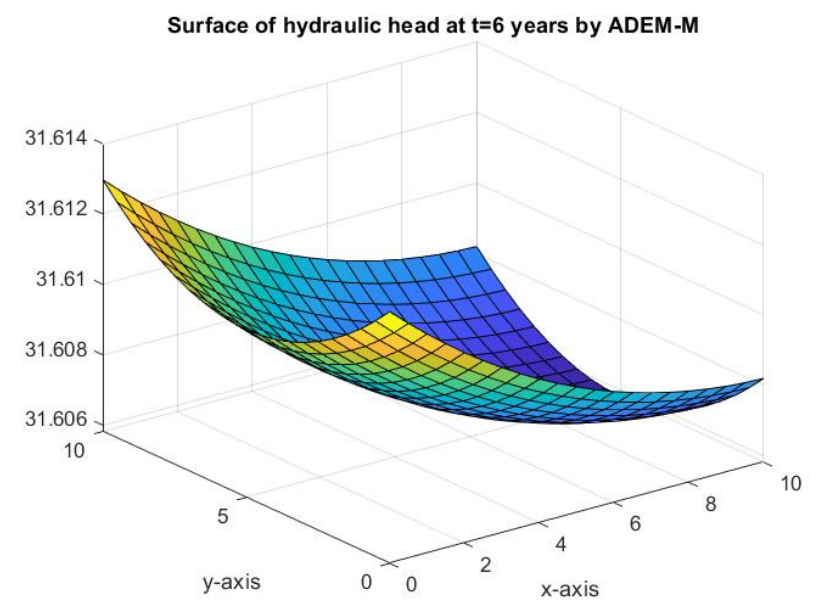

Figure 2. Hydraulic head of the considered area in 6 years in simulation 1

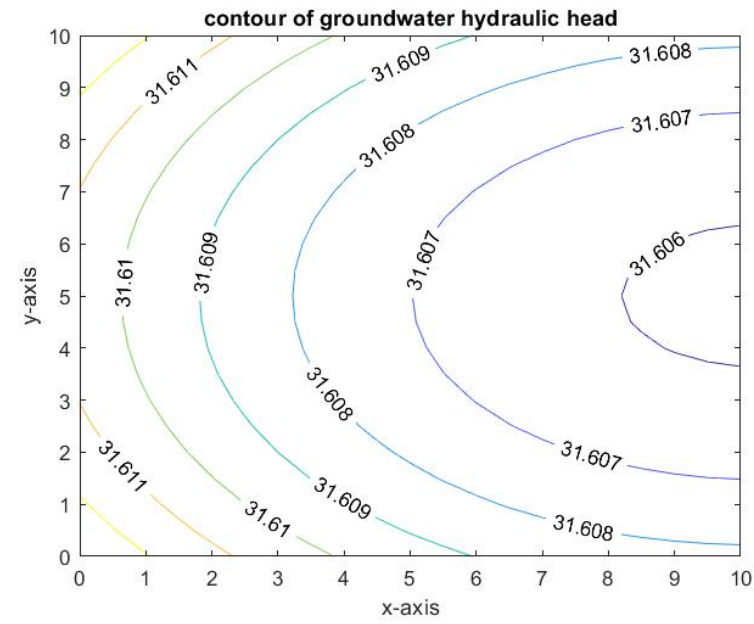

Figure 3. Hydraulic head level at 6 years in simulation 1

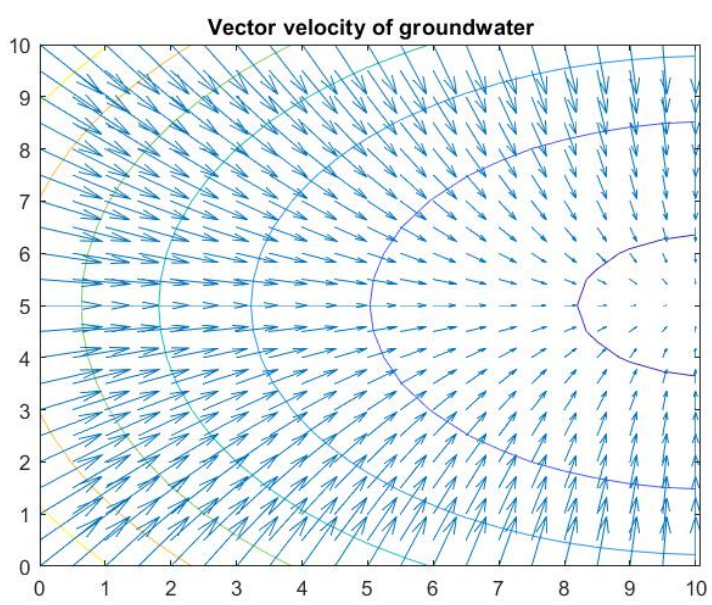

Figure 4. The groundwater flow direction at 6 years in simulation 1

The initial $\psi_{0}=0 \mathrm{~m}$ and boundary conditions $N_{\psi}=S_{\psi}=$ $W_{\psi}=E_{\psi}=0$. The approximate solution as shown in Fig 6 .

Considering diffusion coefficient of nitrite $D_{2 x}=D_{2 y}=$ 
0.00123 and rate of ammonia dispersion $R_{1}=0.00001$. The initial $\xi_{0}=0 \mathrm{~m}$ and boundary conditions $N_{\xi}=S_{\xi}=W_{\xi}=$ $E_{\xi}=0$. The approximate solution as shown in Fig 7.

Considering diffusion coefficient of nitrate $D_{3 x}=D_{3 y}=$ 0.00162 and rate of nitrite dispersion $R_{2}=0.0005$. The initial zet $_{0}=0 \mathrm{~m}$ and boundary conditions $N_{\zeta}=S_{\zeta}=W_{\zeta}=$ $E_{\zeta}=0$. The approximate solution as shown in Fig 8 .

The nitrogen compounds pollutant levels are compared in Fig 9.

\subsection{Numerical simulation of nitrogen dispersion with pumping sources}

The application of nitrogen dispersion models defined grid space $\Delta x=0.5(\mathrm{~m})$ and $\Delta y=0.5(\mathrm{~m})$ time $\Delta t=0.1$ $\left(\mathrm{m}^{2}\right)$, increments in area $10 \times 10$. The first model, considering the hydraulic conductivity in homogeneous aquifer $K=0.15$ (m/day), the specific storage coefficient $S=10$, source function $W=0.1\left(\mathrm{~d} a y^{-1}\right)$, one pumping $W 1=-0.1\left(\mathrm{~d} a y^{-1}\right)$ and two pumping $W 2=0.05\left(\mathrm{~d} a y^{-1}\right)$. The initial $h_{0}=30$ (m) and boundary conditions $N_{h}=0.01, S_{h}=-0.01$, $W_{h}=-0.01$ and $E_{h}=0$. It mean that water flows to north, south and west on $\Omega$ domain. Consider the hydraulic head of groundwater by using the modified alternating direction explicit method approximate solution as shown in Fig 10 - Fig 11.

The second model, input hydraulic head in first model to approximate groundwater velocity in ( 8 ) and ( 9 ) by using CS. The direction of groundwater in consider area as shown in Fig 12

Next, input groundwater to approximate nitrogen dispersion. Organic material is transformed into ammonia, then into nitrite, and lastly into nitrate by using FTCS, considering diffusion coefficient of nitrogen $D_{0 x}=D_{0 y}=0.002$ and source function of pollution through specified $W s=0.001 * W$. The initial $\phi_{0}=1 \mathrm{~m}$ and boundary conditions $N_{\phi}=0.001 N_{h}$, $S_{\phi}=-0.001 S_{h}, W_{\phi}=-0.001 W_{h}$ and $E_{\phi}=0.001 E_{h}$. It mean that water flows to north, south and west on $\Omega$ domain. The approximate solution as shown in Fig 13.

Considering diffusion coefficient of ammonia $D_{1 x}=$ $D_{1 y}=0.0013$ and rate of nitrogen dispersion $R_{0}=0.00005$. The initial $\psi_{0}=0 \mathrm{~m}$ and boundary conditions $N_{\psi}=S_{\psi}=$ $W_{\psi}=E_{\psi}=0$. The approximate solution as shown in Fig 14 .

Considering diffusion coefficient of nitrite $D_{2 x}=D_{2 y}=$ 0.00123 and rate of ammonia dispersion $R_{0}=0.00001$. The initial $\xi_{0}=0 \mathrm{~m}$ and boundary conditions $N_{\xi}=S_{\xi}=W_{\xi}=$ $E_{\xi}=0$. The approximate solution as shown in Fig 15 .

Considering diffusion coefficient of nitrate $D_{3 x}=D_{3 y}=$ 0.00162 and rate of nitrite dispersion $R_{0}=0.0005$. The initial $\zeta_{0}=0 \mathrm{~m}$ and boundary conditions $N_{\zeta}=S_{\zeta}=W_{\zeta}=E_{\zeta}=0$. The approximate solution as shown in Fig 16.

The nitrogen compounds pollutant levels are compared in Fig 17.

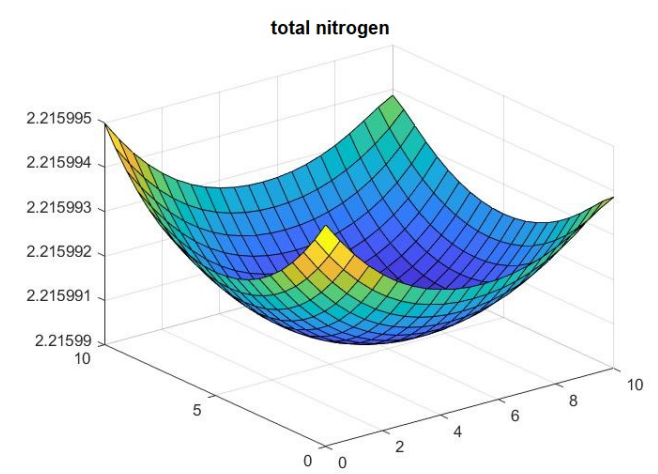

Figure 5. Nitrogen concentration at 6 years in simulation 1

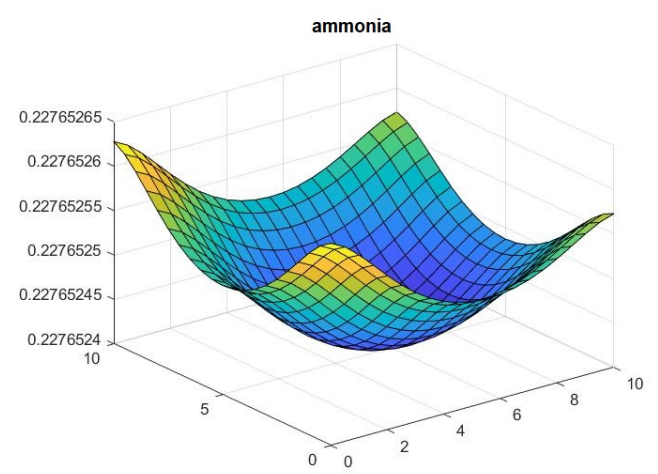

Figure 6. Ammonia concentration at 6 years in simulation 1

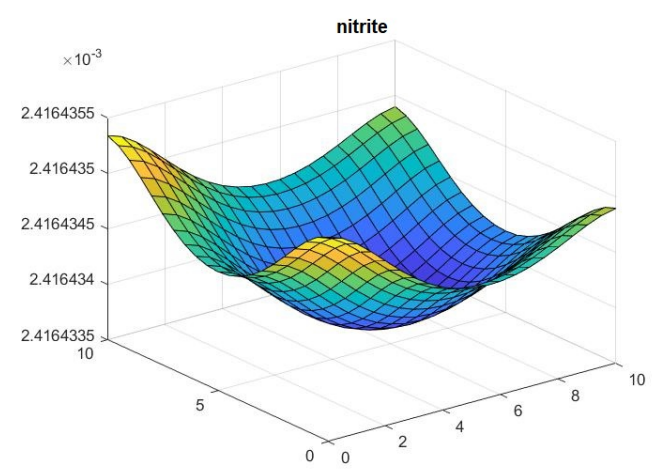

Figure 7. Nitrite concentration at 6 years in simulation 1

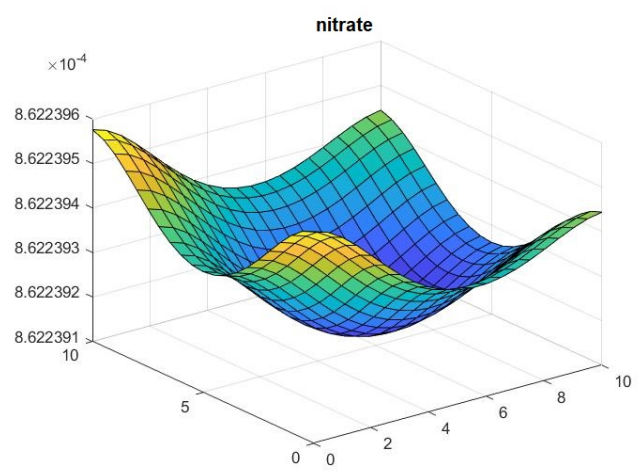

Figure 8. Nitrate concentration at 6 years in simulation 1

\section{Discussion}

In first simulation, we can obtain the hydraulic head in the considered area along 6 years as show in Fig 2-3. The fig- 


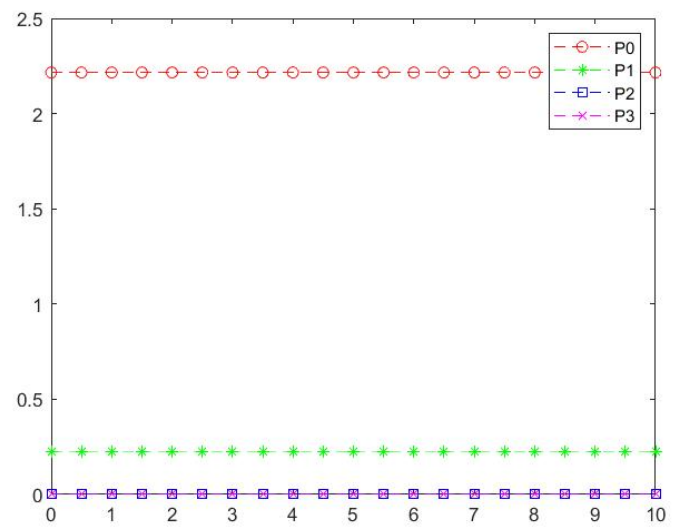

Figure 9. Comparison of nitrogen compound levels along 6 years in simulation 1 with $\mathrm{P} 0=$ total nitrogen, $\mathrm{P} 1=$ ammonai, $\mathrm{P} 2=$ nitrite, $\mathrm{P} 3=$ nitrate

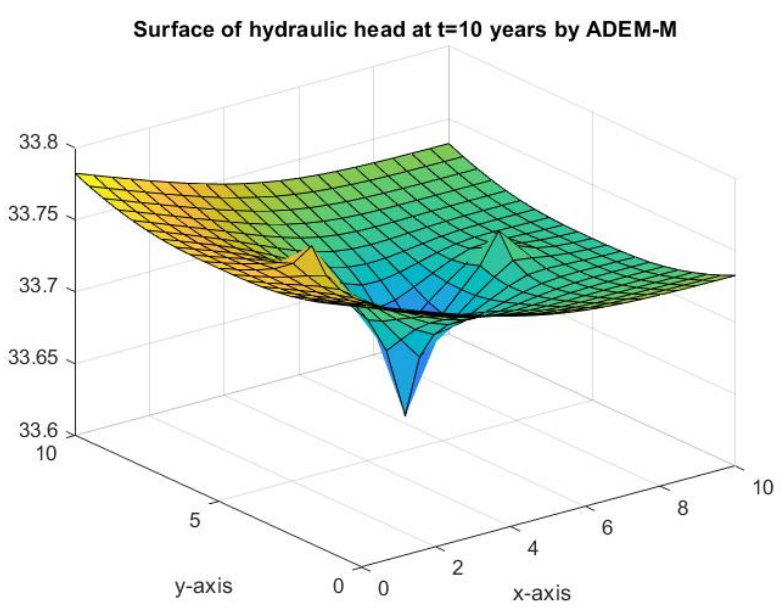

Figure 10. Hydraulic head of the considered area in 10 years in simulation2

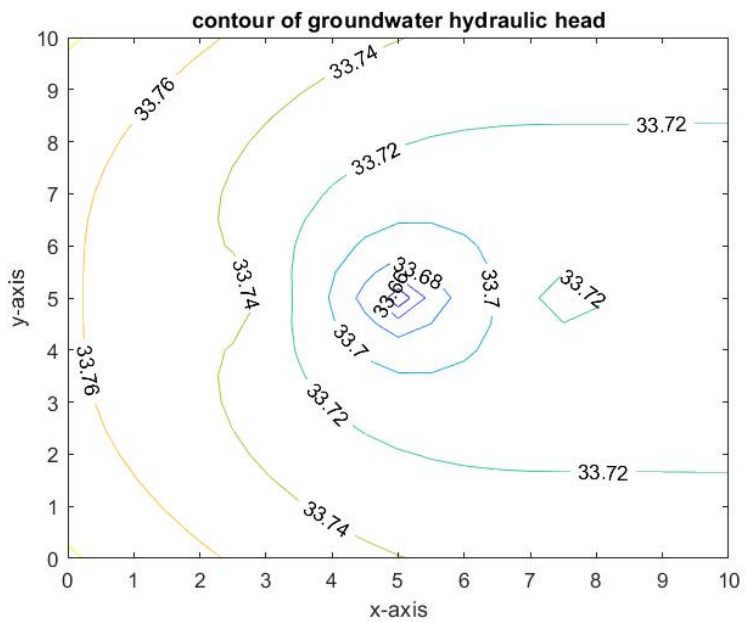

Figure 11. Hydraulic head level at 10 years in simulation 2

ures have shown that the hydraulic head are slowly increasing around areas of water flows in and slowly decreasing around areas of water flows out. The hydraulic head is transformed to be the groundwater flow direction as shown in Fig 4. The

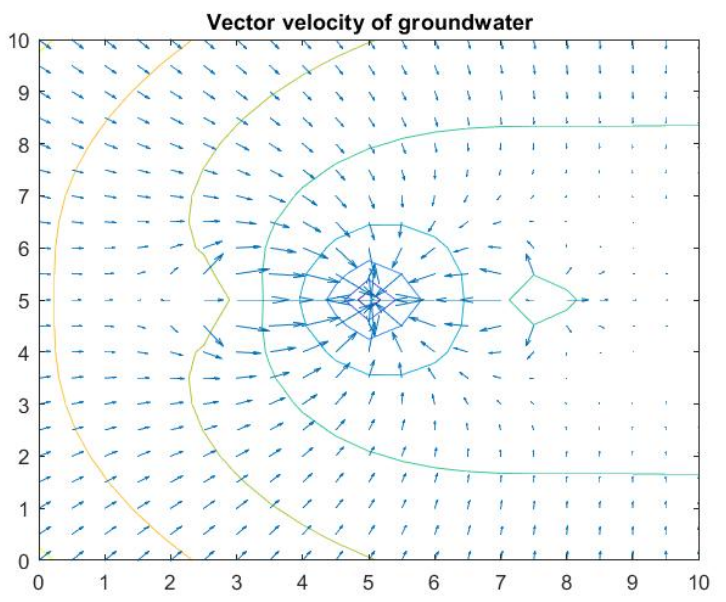

Figure 12. The groundwater flow direction at 10 years in simulation 2

directions have shown that groundwater flow from high to low hydraulic head. They are used to plug into the four nitrogen compound dispersion models. We can measure the total nitrogen, ammonia, nitrite and nitrate groundwater pollutant levels at 6 years as shown in Fig. 5-8 The approximated nitrogen compound is compared in Fig 9 along 6 years. The figures have shown that the amount of that groundwater toxicity changes directly over time and the substance is less than reactant. The second simulation gives more realistic scenario than the first simulation. The simulation is containing the effect of the groundwater pumping station and the groundwater injective stations. The hydraulic head levels are still approximated as shown in Fig 10-11 Similarly, the groundwater direction are also simulated as shown in Fig 12. They are used to plug into the four nitrogen compound dispersion models. We can measure the total nitrogen, ammonia, nitrite and nitrate groundwater pollutant levels at 10 years as shown in Fig. 13-16 The approximated nitrogen compound is compared in Fig 17 along 10 years.

\section{Conclusions}

A two-dimensional groundwater flow model that provides the hydraulic head is introduce. Consequently, a twodimensional groundwater flow velocity model is also introduced. The nitrogen compound dispersion model groundwater pollutant concentration such as that provides the total nitrogen concentration, the ammonia concentration, the nitrite concentration and the nitrate concentration. A modified alternating direction explicit method is used to approximate the hydraulic head model solution. The approximated hydraulic head is plugged into the second model. The centered space finite difference technique is employed to approximate the groundwater flow velocity model solution. Next, the forward time centered space finite difference technique is used to approximate the total nitrogen dispersion model solution, an ammonia dispersion model solution, a nitrite dispersion model solution and a nitrate dispersion model solution, respectively. The nu- 


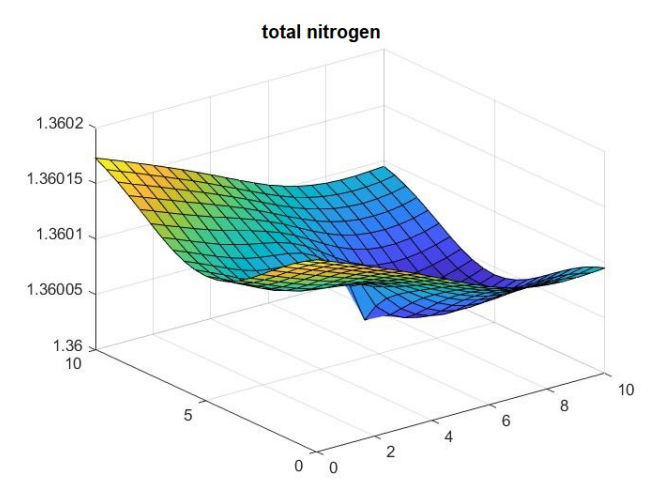

Figure 13. Nitrogen concentration at 10 years in simulation 2

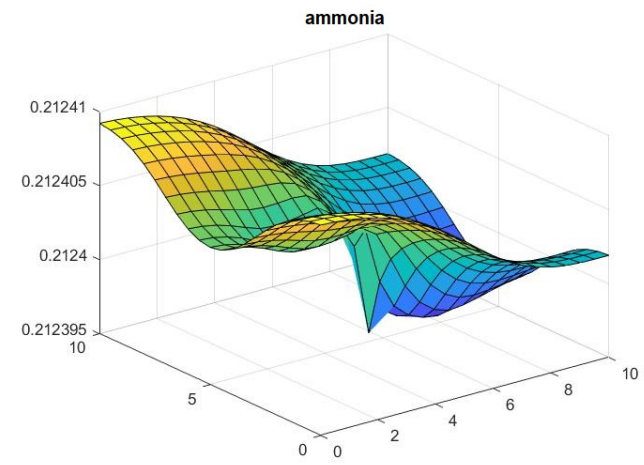

Figure 14. Ammonia concentration at 10 years in simulation 2

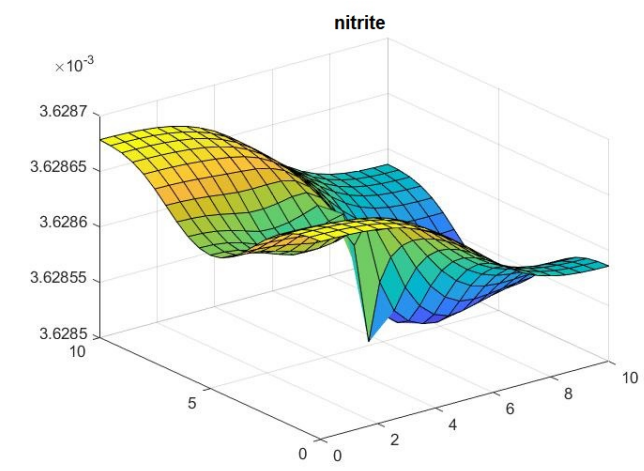

Figure 15. Nitrite concentration at 10 years in simulation 2

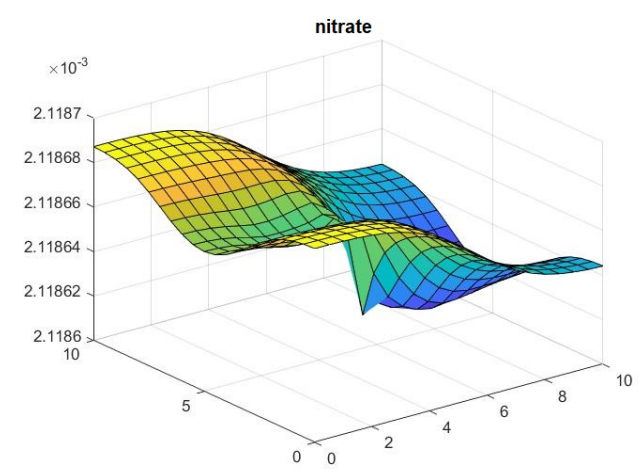

Figure 16. Nitrate concentration at 10 years in simulation 2

merical experiment is tested into two simulations such as numerical simulation of nitrogen dispersion and numerical sim-

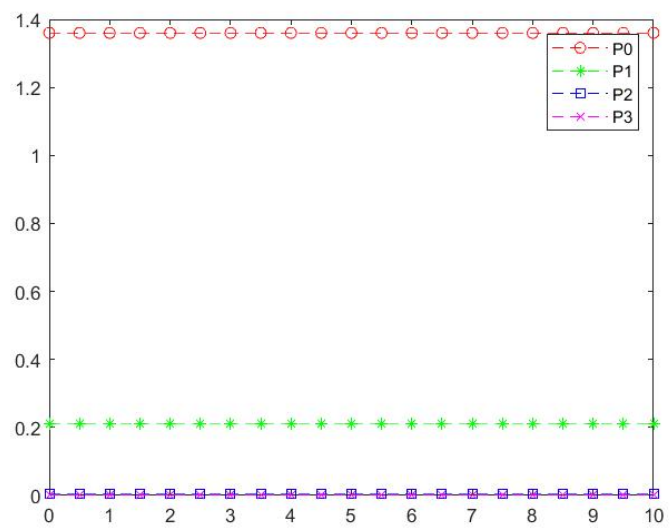

Figure 17. Comparison of nitrogen compound levels along 10 years in simulation 2 with $\mathrm{P} 0=$ total nitrogen, $\mathrm{P} 1=$ ammonai, $\mathrm{P} 2=$ nitrite, $\mathrm{P} 3=$ nitrate

ulation of nitrogen dispersion with pumping sources. We can obtain the hydraulic head, groundwater flow direction, the total nitrogen dispersion model, an ammonia dispersion model, a nitrite dispersion model and a nitrate dispersion model along 10 years when there is groundwater pumping system. The propose model can predict the nitrogen compound pollutant effect and show the highest risk for contamination of groundwater by total nitrogen in a long term when there is the groundwater usage. Because groundwater is an important water sources for longterm storage. Therefore, the controlling nitrogen method is a primary key to controlling the source of nitrogen compound pollutant. Especially, the groundwater contamination do effect to the nitrogen cycle rate increasing.

\section{Acknowledgements}

This research is supported by the Centre of Excellence in Mathematics, the Commission on Higher Education, Thailand.

\section{REFERENCES}

[1] Majumdar D. (2003). The Blue Baby Syndrome Nitrate Poisoning in Humans, Resonance, 8(10), 20-30.

[2] Song P., Wu L., and Guan W. (2015). Dietary Nitrates, Nitrites, and Nitrosamines Intake and the Risk of Gastric Cancer: A Meta-Analysis. Nutrients , 7(12), 9872-9895. doi:10.3390/nu7125505

[3] Linsha M, Liang H, Xiaoyu F and Songlin W. (2018). Nitrate and Nitrite in Health and Disease, Aging and Disease, 9(5), 938-945.

[4] Yamashita N., Sugio S. (2000) Numerical Simulation of Nitrate Transport with Unsaturated Flow Condition in Volcanic Soils. In: Sato K., Iwasa Y. (eds) Groundwater Updates. Springer, Tokyo.

[5] Dehghan M., (2004). Weighted finite difference techniques for the one-dimensional advection-diffusion equation, Applied Mathematics and Computation, 147(2), 307-319. 
[6] Gardenas, A I , Hopmans, J W , Hanson, B R , and Simunek J. (2005). Two-dimensional modeling of nitrate leaching for various fertigation scenarios under micro-irrigation.

[7] Zhiqiang D, Shaowei S, Daniel D G, (2011). Modeling nitratenitrogen removal process in first-flush reactor for stormwater treatment, Bioprocess Biosyst Eng, 35(6), 865-874.

[8] Kraychang W and Pochai N, (2016), Implicit Finite Difference Simulation of Water Pollution Control in a Connected Reservoir System, International Journal of Applied Mathematics 46 (1).

[9] Suebyat K and Pochai N, (2018), Numerical Simulation for a Three-Dimensional Air Pollution Measurement Model in a Heavy Traffic Area under the Bangkok Sky Train Platform, Abstract and Applied Analysis.
[10] Brian O. Nitrates and Nitrites in Drinking Water Groundwater and Surface Waters, Water Research Center, https://waterresearch.net/index.php/nitrate.

[11] Pochai N.(2011). A Numerical Treatment of nondimensional form of water quality model in a nonuniform flow stream using saulyev scheme, Hindawi, Mathematical Problems in engineering.

[12] Pongnu N, Pochai N. (2017). Numerical simulation of groundwater measurement using alternating direction methods, Journal of Interdisciplinary Mathematics, 20(2), 513-541.

[13] Joshua H. Viers et al. (2012). Nitrogen Sources and Loading to Groundwater. Center for Watershed Sciences: University of California, Davis. http://groundwaternitrate.ucdavis.edu. 